Check for updates

Cite this: RSC Adv., 2017, 7, 25398

\title{
Scaling the effect of hydrophobic chain length on gene transfer properties of di-alkyl, di-hydroxy ethylammonium chloride based cationic amphiphiles $\uparrow$
}

\author{
Ankita A. Hiwale, $\$^{\mathrm{a}}$ Chandrashekhar Voshavar, $\$^{\mathrm{b}}$ Priya Dharmalingam, ${ }^{\mathrm{c}}$ \\ Ashish Dhayani, ${ }^{a}$ Rajesh Mukthavaram, ${ }^{d}$ Rasajna Nadella, ${ }^{c}$ Omprakash Sunnapu, ${ }^{e}$ \\ Sivaraman Gandhi, ${ }^{a}$ V. G. M. Naidu, ${ }^{e}$ Arabinda Chaudhuri, (D) Srujan Marepally (D) *ac \\ and Praveen Kumar Vemula (D) *a
}

\begin{abstract}
The success of gene therapy critically depends on the availability of efficient transfection vectors. Cationic lipids are the most widely studied non-viral vectors. The molecular architecture of the cationic lipid determines its transfection efficiency. Variations in alkyl chain lengths of lipids influence self-assembly and liposomal fusion with the cell membrane. These factors determine the transfection ability of the lipid. Thus, to probe the effect of asymmetry in hydrophobic chains on transfection efficiency, we designed and synthesized a series of cationic lipids by systematically varying one of the two alkyl chains linked to the quaternary nitrogen centre from C18 to C10 and keeping the other alkyl C18 chain constant (Lip1818-Lip1810). Transfection studies in multiple cultured mammalian cells (CHO, B16F10 and HeLa) revealed that the lipids with $\mathrm{C} 18: \mathrm{C} 14$ and $\mathrm{C} 18: \mathrm{C} 12$ alkyl chains (Lip1814 \& Lip1812) showed 20-30\% higher transfection efficacies than their counterparts at $2: 1$ and $4: 1$ lipid to pDNA charge ratios. Cryo-transmission electron images showed unilamellar vesicle structures for the liposomes of lipids. Mechanistic studies involving Small Angle X-ray Scattering (SAXS) revealed that asymmetry in the hydrophobic region has a significant impact on liposomal fusion with the plasma membrane model. Collectively, these findings demonstrate that chain length asymmetry in the hydrophobic region of cationic lipids has an important role in their liposome-DNA interactions at optimal $2: 1$ and $4: 1$ lipid to pDNA charge ratios, which in turn modulates their gene transfer properties.
\end{abstract}

\author{
Received 23rd February 2017 \\ Accepted 28th April 2017 \\ DOI: $10.1039 / c 7 r a 02271 a$ \\ rsc.li/rsc-advances
}

\section{Introduction}

Intracellular delivery of nucleic acids is one of the promising strategies for the development of therapeutic modalities for treating genetic diseases. ${ }^{1-3}$ Use of cationic lipids has received significant interest to deliver nucleic acids to the specific sites in

anstitute for Stem Cell Biology and Regenerative Medicine (inStem), GKVK-post, Bellary Road, Bengaluru 560065, India. E-mail: Praveenv@instem.res.in

${ }^{b}$ BioSatva Technologies, Golnaka, Hyderabad 500013, India

${ }^{c}$ Centre for Stem Cell Research, Christian Medical College Campus, Bagayam, Vellore 632002, India.E-mail: Srujankm@cmcvellore.ac.in

${ }^{d}$ Translational Neuro-oncology Laboratories, Moores Cancer Center, University of California San Diego, La Jolla, CA, USA

${ }^{e}$ National Institute for Pharmaceutical Education and Research, Balanagar, Hyderabad 500018, India

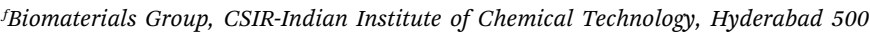
007, India

$\dagger$ Electronic supplementary information (ESI) available: ${ }^{1} \mathrm{H} \&{ }^{13} \mathrm{C}$ NMR and ESI-MS spectral characterizations for all the cationic amphiphiles, RP-HPLC chromatograms for target lipids, size and surface potential of liposome \& lipoplexes, cytotoxicity \& transfection data. See DOI: 10.1039/c7ra02271a

$\$$ Equally contributing authors.

a targeted manner. ${ }^{4,5}$ The success of nucleic acid-based therapies critically depends on the efficiency of the carrier. ${ }^{6}$ Towards developing efficient liposomal systems, structure-activity studies varying the hydrophilic head group, linker functionality and hydrophobic tail of cationic lipids have been performed..$^{7,8}$ However, these studies indicated a general trend of increased transfection activity as the saturated aliphatic chains increased from C14 to C18. ${ }^{9}$ Prior findings demonstrated that alkyl chain length had a major role in determining the rigidity of the liposomes. ${ }^{10,11}$ Longer alkyl chains such as C18 imparts rigidity due to its higher hydrophobicity and ordered packing, while shorter chains such as $\mathrm{C} 10-\mathrm{C} 14$ increases the membrane fluidity. ${ }^{9}$ Hence, finding the optimal chain length to achieve higher transfection efficiency is a challenging task. ${ }^{12}$ However, the possible best results can be achieved with a balance between hydrophilic moiety and hydrophobic chain lengths along with a spacer, if any. ${ }^{13}$ Prior findings, including our own, demonstrated that cationic lipids with the shorter aliphatic chains (C12 \& C14) found to be more efficient under in vitro conditions while longer aliphatic chains (C16 \& C18) are active for in vivo nucleic acid delivery. ${ }^{14}$ However, it is difficult to generalize the 
phenomenon as other factors such as head group and linker play key roles in determining the transfection efficacies both in vitro and in vivo. Understanding the effect of variation in the hydrophobic region of cationic amphiphiles is critical for the development of efficient transfection reagents. ${ }^{15-21}$ First developments of cationic lipids were reported with identical hydrophobic alkyl chains (symmetric chains). ${ }^{13,22}$ Zhi et al., demonstrated that transfection could be enhanced by doping two cationic lipids with the same head group and symmetric chains with varying lengths. ${ }^{17,23}$ Such improved transfection could be due to enhanced inter-membrane mixing of different hydrophobic chains during endocytosis or early endosomal escape of nucleic acids. ${ }^{16}$ Prior studies, including our own, convincingly demonstrated that the lipids with asymmetry in the hydrophobic region had higher transfection properties than their symmetric counterparts. ${ }^{\mathbf{1 6 1 9 , 2 0 , 2 4}}$ Nantz group demonstrated that the hydrophobic chain asymmetry varying from myristoyl to lauroyl (2 carbons variation) in the liposomal system significantly enhanced the in vivo transfection efficiency of non-glyceryl based lipids. ${ }^{19}$ However, to the best of our knowledge, in-depth structure-activity investigations were not yet reported addressing the effect in non-glyceryl based lipids or lipids without linkers so far. More importantly, the role of different chain lengths in liposome and DNA interactions with varying charge ratios are not clearly understood. Given the strong modulation effects of asymmetry in the chain lengths on transfection efficacies, we planned to explore the role of variation in hydrophobic chains with a systematic structure-efficiency investigation. To this end, we have used one of our previously reported efficient transfection reagent, DODEAC ( $N, N$-di- $n$-octadecyl- $N, N$-dihydroxyethyl ammonium chloride) as a symmetric core/model lipid. ${ }^{25}$ This cationic lipid contains two C18 chains attached to a quaternary nitrogen-containing two hydroxyl ethylene head groups.

To scale the effect of asymmetry within the hydrophobic region of our DODEAC cationic amphiphile, we have synthesized a series of cationic amphiphiles with C18 as a common chain in all the cationic amphiphiles, while varying the other chain from C16-C10 (Fig. 1). We have used DODEAC (with two C18 chains) as control symmetric lipid. Transfection studies in cultured mammalian cell lines showed 20-30\% higher transfection efficiency for Lip1814 and Lip1812 in the series. Herein, we present systematic structure-activity investigation on the role of asymmetric hydrophobic chains within a di-alkyl, di-

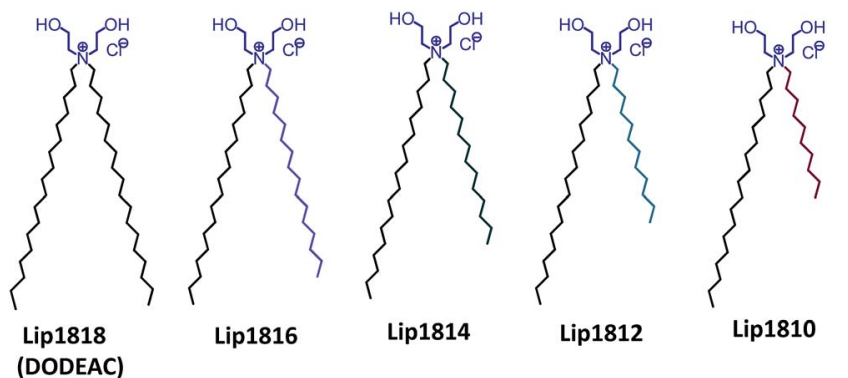

Fig. 1 Chemical structures of cationic amphiphiles with varying chain lengths. Lipids (Lip1816-Lip1810) were synthesized with varying alkyl chain lengths in the hydrophobic region of a previously reported transfection reagent 'DODEAC'. hydroxy ethyl ammonium chloride based cationic amphiphile in influencing transfection activity. Our study includes design, synthesis of lipid series (Lip1818-Lip1810, Fig. 1), physicochemical characterization and transfection studies using liposomes of synthesized lipids in cultured mammalian cell lines.

\section{Results and discussion}

A number of structure-activity investigations have been carried out on the core template/moieties of a cationic amphiphile with varying head groups, charges, spacer effects and hydrophobic cores for enhancing transfection efficiency. ${ }^{22}$ However, chain length alterations within the hydrophobic core of cationic amphiphiles were not well understood. Such modification studies on the hydrophobic core involved the use of either different alkyl chain lengths or steroidal backbones such as cholesterol and bile acids. To this end, we wanted to evaluate the effect of length of asymmetric chains within the hydrophobic core of a successful transfection reagent, DODEAC. ${ }^{25}$ We hypothesized that balanced asymmetric chain lengths within di-alkyl, di-hydroxy ethyl ammonium chloride based cationic amphiphiles would cause membrane perturbation resulting in enhanced endocytosis and ultimately leading to a higher transfection activity.

\section{Synthesis of lipids (Lip1818-Lip1810)}

To begin with, we have synthesized a series of lipids (Fig. 1) with variation in one of the two alkyl chains attached to a quaternary nitrogen centre of a cationic lipid, DODEAC. Each lipid possessed one C18 alkyl chain while varying the length of second alkyl chain from $\mathrm{C} 18$ to $\mathrm{C} 10$ in the hydrophobic region of the lipid. All the lipids were synthesized following 2-step reactions. Initially, individual asymmetric chain containing secondary amines were prepared by $N$-alkylation of stearyl amine using individual alkyl bromides (C16-C10) along with symmetric C18 bromide. All the secondary amines were subjected for quaternization using 2chloroethanol to yield final compounds (Lip1818-Lip1810, Fig. 2). All the lipids were purified by re-crystallization and characterized using NMR $\left({ }^{1} \mathrm{H} \&{ }^{13} \mathrm{C}\right)$ and ESI-MS. For both secondary amine intermediates (1A-5A, Fig. 2) and final lipids (Lip1818-Lip1810), there was no significant variation in the yields with a decrease in the chain length.

\section{Physico-chemical characterization}

One of the major issues in the transfections is the stability of lipoplexes and their membrane interaction, which plays a key role in cellular uptake and strongly modulate the transfection efficacies. Such modulating factors are likely to depend on the size and global surface charges of lipoplexes. Self-assembly of cationic lipids (Lip1818-Lip1810, Fig. 1) produced unilamellar vesicles that were characterized using dynamic light scattering (DLS) and cryo-TEM. Liposomes of these lipids showed hydrodynamic diameter in the range of 125 to $150 \mathrm{~nm}$ (Table S1, ESI $\dagger$ ), while zeta potentials were within +45 to +49 except Lip1810 which showed +35.7 (Table S1, ESI $\dagger$ ). Size \& surface potential measurements of liposomes did not show any difference among lipids, Lip1818-Lip1810 possibly due to single 
Synthesis of cationic Lipids $1-5$ with varying one alkyl chain

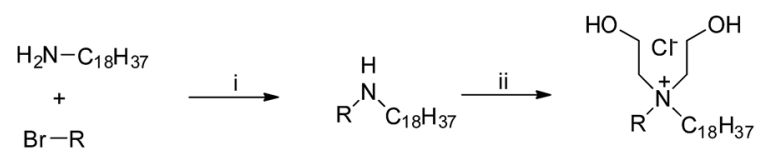

$1 \mathrm{~A}-5 \mathrm{~A}$

Lipids 1 - 5

\begin{abstract}
Lipid $1 \mathrm{R}=\mathrm{n}-\mathrm{C}_{10} \mathrm{H}_{21}: 71 \%$
Lipid $2 \mathrm{R}=\mathrm{n}-\mathrm{C}_{12} \mathrm{H}_{25}: 69 \%$

Lipid $3 \mathrm{R}=\mathrm{n}-\mathrm{C}_{14} \mathrm{H}_{29}: 70 \%$

Lipid $4 \mathrm{R}=\mathrm{n}-\mathrm{C}_{16} \mathrm{H}_{33}: 67 \%$

Lipid $5 \mathrm{R}=\mathrm{n}-\mathrm{C}_{18} \mathrm{H}_{37}: 65 \%$
\end{abstract}

Reagents: i) $\mathrm{K}_{2} \mathrm{CO}_{3}, \mathrm{DMSO}, 80^{\circ} \mathrm{C}, 12 \mathrm{~h}$; ii) 2-Chloroethanol, $80^{\circ} \mathrm{C}, 24 \mathrm{~h}$.

Fig. 2 Synthesis of cationic lipids, Lip1818-Lip1810.

positive charge in all five lipids. Furthermore, liposomes made from all five lipids were complexed with plasmid DNA (pDNA), and size \& zeta potentials of the resulting lipoplexes were measured (Tables S2 and S3, ESI $\dagger$ ). Data suggests that lipoplexes (lipid-DNA complexes) have higher size (400-1900 nm) compared to liposomes (125-150 nm). Interestingly, lipid/DNA charge ratio seems to have a significant effect on size and potentials of lipoplexes. Decreasing lipid/DNA charge ratio from $8: 1$ to $1: 1$ increased the size of lipoplexes (Table S2, ESI $\dagger$ ) while zeta potential has decreased (Table S3, ESI $\dagger$ ). Surface potentials of lipoplexes at $8: 1-4: 1$ lipid : DNA charge ratios are positive due to higher lipid ratio. While, at lower charge ratios $2: 1-1: 1$ varied with a low positive charge to mostly negative charge for all the lipids (Table S3, ESI $\dagger$ ). Overall, data from global surface charges and potentials indicate that there is no clear correlation with transfection efficiencies of lipids, Lip1818-Lip1810. It also rules out the possibility of their major role in influencing the transfection efficacies. Next, we measured the DNA binding ability for lipids Lip1818-Lip1810 across lipid : DNA charge ratios $8: 1-1: 1$ to understand whether any difference in the lipid DNA interactions using conventional agarose gel retardation assay. Liposomes of all the lipids were complexed with pDNA and subjected to gel retardation assay in 1\% agarose gel with ethidium bromide as intercalating agent. The lipid : DNA ratios are indicated at the top of each lane.

Results from gel-retardation assay showed that DNA was completely complexed with the liposomes of Lip1818-Lip1810 from lipid/DNA charge ratios, $8: 1$ to $2: 1$ while $1: 1$ had some unbound DNA (Fig. 3). The conventional gel retardation assay indicates strong lipid : DNA binding interactions for all the lipids, reveals that the hydrophobic chain length variation has no role in determining the lipid-DNA interactions. Following DLS measurements and DNA binding studies, we have employed the cryo-TEM technique to observe the structural characteristics of liposomes of Lip1818-Lip1810. Cryo-TEM images showed that liposomes of individual lipids possess similar structural and size patterns $(\sim 100-150 \mathrm{~nm})$ revealing their unilamellar nature (Fig. 4). Physico-chemical data for

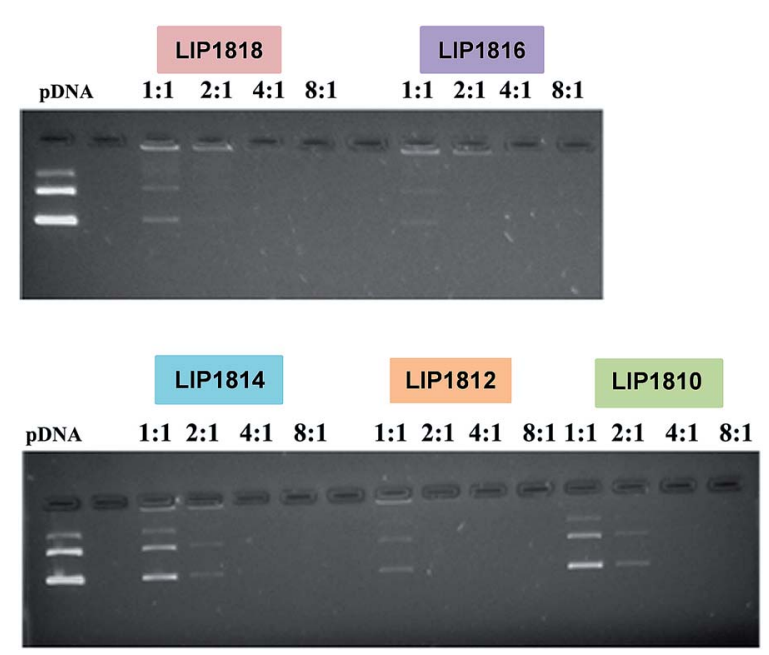

Fig. 3 Electrophoretic gel patterns for lipid-associated DNA in gel retardation assay for lipids (Lip1818-Lip1810).
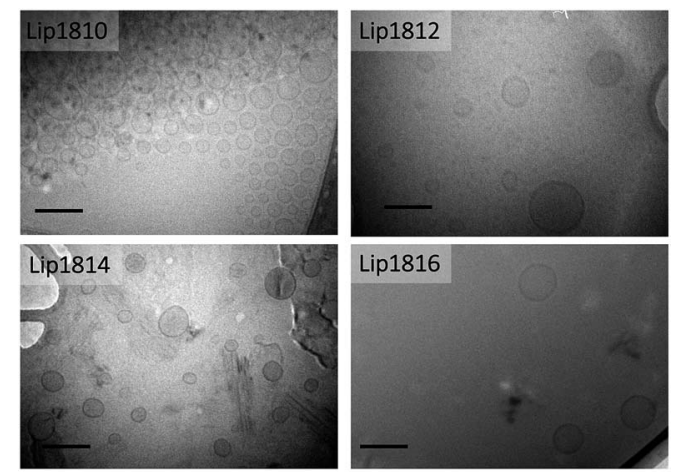

Lip1816
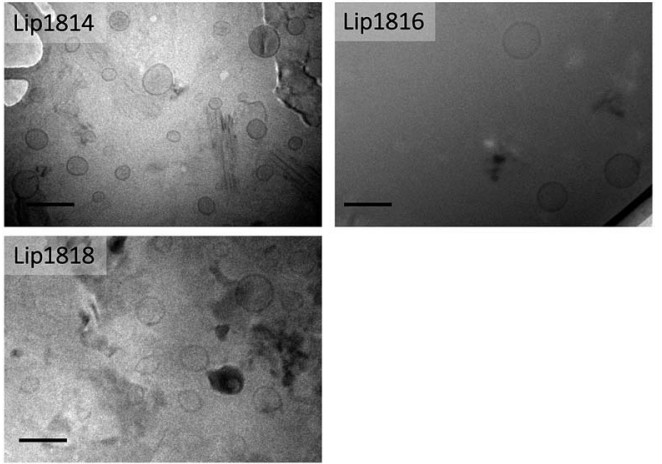

Fig. 4 Cryo-transmission electron microscopic images of liposomes of lipids (Lip1818-Lip1810) (scale $0.1 \mu \mathrm{m}$ ).

liposomes and lipoplexes of Lip1818-Lip1810 obtained from DNA binding, DLS studies and cryo-TEM collectively demonstrated that all the lipids formed unilamellar liposomes and showed efficient complexation with pDNA.

\section{Cytotoxicity assay}

MTT-based colorimetric cell viability assay was performed in representative HeLa cells with lipoplexes of lipids (Lip1818Lip1810) and plasmid DNA for $24 \mathrm{~h}$. Results showed high percent cell viabilities (>75\%) for lipids Lip1818-Lip1810 within all the lipid : DNA charge ratios (Fig. S1, ESI $\dagger$ ). HeLa cells treated with lipoplexes of lipids Lip1818-Lip1810 at 1:1 and 2 : 1 charge ratio revealed remarkably high cell viabilities $(>95 \%$ and $>90 \%$ for $1: 1 \& 2: 1$ charge ratios, respectively). Percent cell viabilities at $4: 1$ and $8: 1$ charge ratios of lipids, Lip1818- 

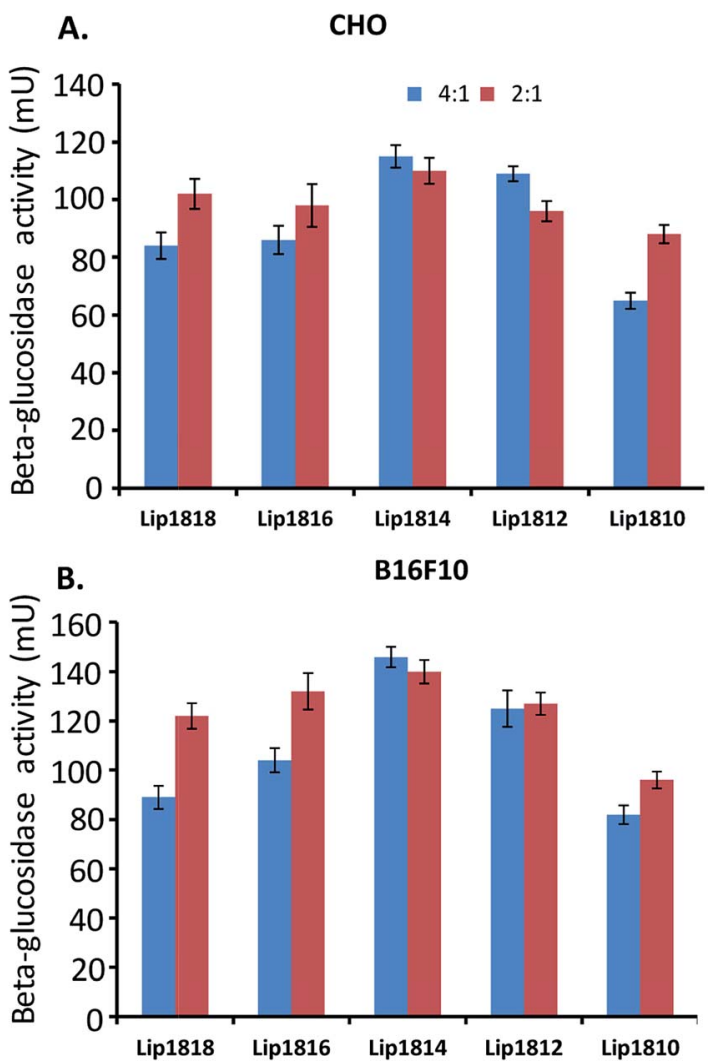

Fig. 5 In vitro transfection studies of lipids (Lip1818-Lip1810). Transfection efficiencies of lipids were evaluated in $\mathrm{CHO}$ and B16F10 cell lines at lipid: DNA charge ratio of $2: 1$ and $4: 1$. Absorption at $405 \mathrm{~nm}$ was converted to $\beta$-galactosidase units using a calibration curve constructed with pure commercial $\beta$-galactosidase enzyme. Units of $\beta$-galactosidase activity were plotted against the lipids. The transfection values shown are the average of triplicate experiments performed with 4-6 data points. $\$$

Lip1810 were found to be low within the range of $90-75 \%$ as depicted in Fig. S1, ESI. $\dagger$ Since all the lipids showed comparable cell viability profiles across varying lipid : DNA charge ratios, the enhanced and/or compromised transfection efficacies of individual lipids Lip1818-Lip1810 cannot be attributed to their cytotoxicity.

\section{Transfection studies}

In vitro transfection efficacies were evaluated to compare the enhancement in their activities due to change in the hydrophobic chain lengths for the series of lipids, Lip1818-Lip1810 in cultured animal cell lines, CHO and B16F10 using pCMV-SPORT- $\beta$-gal plasmid DNA encoding a reporter gene/enzyme ' $\beta$-galactosidase'. Transfection studies in both cell lines i.e. CHO \& B16F10 showed an interesting trend of transfection profiles among lipids Lip1818-Lip1810 with an inverted ' $V$ ' curve (Fig. 5).

$\S$ Statistical analysis: Data in individual graphs is represented as the mean \pm SD of obtained values from each experiment. Data from each group were compared with other groups within the graph using Student $t$ test. $p<0.05$ was considered as significant.
We found that transfection efficiencies increased as the chain length decreased from C18 to C14 i.e. from Lip1818 to Lip1814 followed by decrease in the transfection with further reduction in the chain lengths, $\mathrm{C} 12$ to $\mathrm{C} 10$ i.e. Lip1812 to Lip1810 in all the lipid : DNA charge ratios except $8: 1$ (Fig. 5 and S2, ESI $\dagger$ ). At $8: 1$ charge ratio, we observed a slight increase in the transfection from Lip1818 to Lip1816 followed by decrease in the transfection (Fig. S2, ESI†). In CHO cells, highest transfection was observed at $4: 1$ lipid : DNA charge ratio for all the lipids (Fig. 5A). In this, Lip1818 \& Lip1816 showed comparatively similar transfection efficacy (with $\sim 85 \mathrm{mU}$ of $\beta$ galactosidase activity) while Lip1814 showed increased activity of $\sim 120$ mU. Lip1812 \& Lip1810 showed $\sim 110$ and $\sim 65 \mathrm{mU}$ of activity respectively. Similar profiles were observed in B16F10 cells at $4: 1$ ratio which showed the highest transfection among all the lipid : DNA charge ratios. Lip1818 showed $\sim 90 \mathrm{mU}$ and Lip1816 exhibited slightly increased activity with $\sim 105 \mathrm{mU}$. However, Lip1814 showed high transfection with $\sim 145 \mathrm{mU}$ while Lip1812 \& Lip1810 showed $\sim 125$ and $\sim 80$ mU of activity respectively (Fig. 5B). Results from transfection studies revealed that the asymmetry has a pattern at $4: 1 \& 2: 1$ lipid to DNA charge ratio in both CHO \& B16F10 cells. In summary, among 5 lipids, Lip1814 and Lip1812 found to be effective in delivering genes compared to Lip1818, Lip1816 and Lip1810 in multiple cultured cell lines. This demonstrated that optimal variation of alkyl chain lengths in di-alkyl, di-hydroxy ethylammonium chloride based cationic amphiphiles are 1814 and 1812. These results suggest that the optimal chain length variation in the hydrophobic core of the lipids is $18: 14$ and $18: 12$, a further increase in the alkyl chain length variations leads to a decrease in the transfection activity that we found in Lip1810 (Fig. 5).

\section{Cellular uptake and expression studies}

Following the transfection experiments, we have performed cellular uptake and expression studies by microscopy imaging at lipid : DNA charge ratio of $4: 1 \& 2: 1$ in representative HeLa cells. To this end, we have transfected HeLa cells with lipoplexes of lipids Lip1818-Lip1810 and pa5GFP plasmid DNA encoding Green Fluorescent Protein (GFP) (Fig. 6). Results from imaging studies are in-line with the transfection data obtained in CHO \& B16F10 cells (compare Fig. 5 with Fig. 6). HeLa cells showed high GFP expression for Lip1814 and Lip1812 followed by Lip1816 \& Lip1810. Lip1818 showed the least fluorescence among all the lipids (Fig. 6A and B). Imaging data obtained is consistent with the transfection data.

\section{SAXS studies}

Interaction of liposomes with cellular membrane plays a key role in the cellular uptake of lipoplexes (lipid-DNA complexes). Liposomes are known to perturb the cell membranes inducing spontaneous curvature to facilitate the endocytosis. ${ }^{26,27}$ The closer arrangement of the individual lipids imparts rigidity to the liposome that in turn affects membrane perturbation properties. To gain insight on the role of the hydrophobic core of each lipid (Lip1818-Lip1810) with the cell membrane, we employed Small Angle X-ray Scattering (SAXS) technique. ${ }^{28}$ In this experiment, 
A

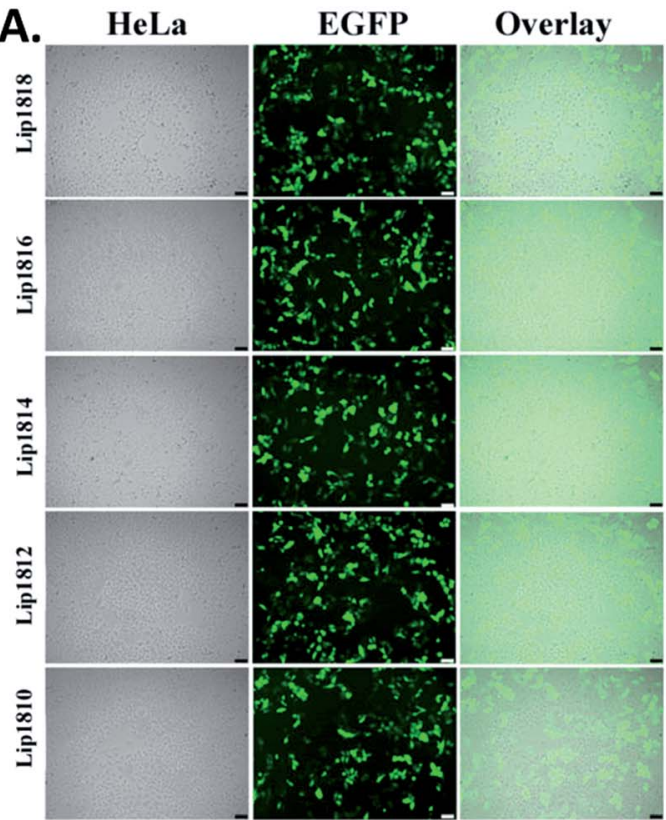

B

\section{B}

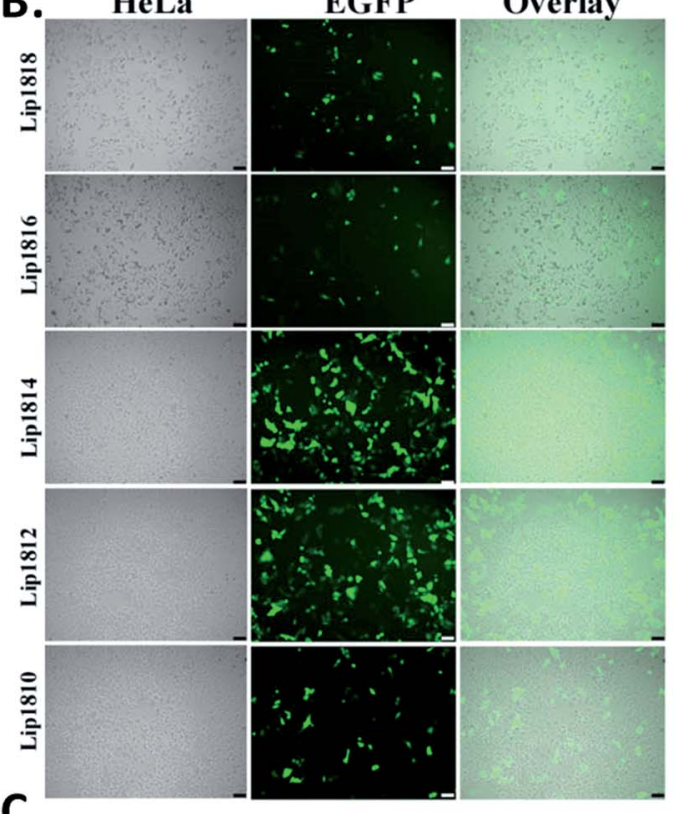

C.

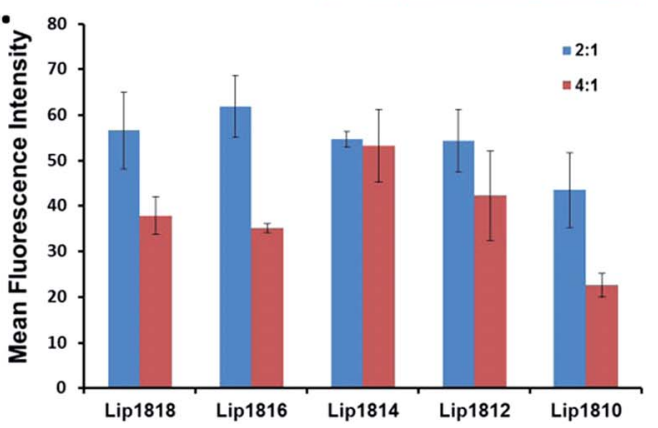

Fig. 6 Cellular uptake and expression studies for lipids (Lip1818Lip1810) in HeLa cells. Microscopic images of the study (A \& B) and quantification of their fluorescence for HeLa cells (C). Cells were transfected with lipoplexes of lipids (Lip1818-Lip1810) and pa5GFP at lipid: DNA charge ratios of $4: 1$ (A) and $2: 1$ (B). a model membrane was prepared with DOPC, DOPE, DOPS and cholesterol at $45: 20: 20: 15$, w/w ratio. Liposomes of each lipid were combined with the model membrane at $1: 1$ molar ratio at $25{ }^{\circ} \mathrm{C}$ was used to record the scattering patterns. SAXS diagrams show a lamellar arrangement for lipids, Lip1818-Lip1810 alone. Scattering curves obtained from SAXS experiment shows perturbation of model membrane with liposomes of lipids Lip1818Lip1810 (Fig. S3, ESI $\dagger$ ). Although it is not clear, we observed sequential variation in the lamellar phase (phase transition) for lipids, Lip1818-Lip1810 with membrane model (Fig. S3, ESI $\dagger$ ). However, due to irregular spacing/peaks in the SAXS patterns, it is hard to identify and assign the transitioned phase. The degree of variation in phase transitions for Lip1816, Lip1814 \& Lip1812 is more prominent in the SAXS diagrams than for Lip1818 \& Lip1810. In addition, liposomes of lipids, Lip1816, Lip1814 \& Lip1812 exhibit strong electrostatic interactions over liposomes of lipids, Lip1818 \& Lip1810 as revealed by their larger variation in the observed intensities ( $Y$-axis in Fig. S3, ESI $\dagger$ ). However, further scattering measurements on the interaction of lipoplexes (lipidDNA complex) of lipids Lip1818-Lip1810 with model biomembrane might shed more light on their membrane perturbation and cellular internalization.

\section{Conclusion}

The role of asymmetric chain lengths within the hydrophobic core of cationic lipids in influencing transfection efficacies was evaluated in the present investigation. We designed and synthesized a series of cationic lipids by varying one of the two alkyl chains linked to quaternary nitrogen centre from C10 to C16 and keeping the other alkyl chain C18 as constant. All the lipids, Lip1818-Lip1810 showed significant transfection efficiencies in delivering reporter genes into multiple cultured mammalian cells including CHO, B16F10 and HeLa. Interestingly, Lip1814 and Lip1812 (with 18, 14 \& 18, 12 alkyl chains respectively) showed higher transfections than the other three lipids at $4: 1$ and $2: 1$ lipid to DNA charge ratio. The transfection profiles revealed that the interaction among lipids with DNA varied with a variation in the asymmetry. Dynamic laser light scattering studies revealed that no significant size difference among the liposomes of lipids, Lip1818-Lip1810. However, liposomes of Lip1814 and Lip1812 exhibited strong DNA condensation at $4: 1$ lipid to DNA charge ratio. These findings were further confirmed by SAXS experiments. Thus, cumulative results suggest that the varying chain length asymmetry within the hydrophobic core of cationic amphiphile has an important role in DNA interaction of its liposome, in turn, affects the transfection properties. Collectively, the present findings further enriched our understanding of the lipid asymmetry and its superior influence on DNA binding properties, consequently determining/enhancing the gene transfer efficacies.

\section{Materials and methods}

\section{General procedures and reagents}

${ }^{1} \mathrm{H} \&{ }^{13} \mathrm{C}$ NMR spectra were recorded on an AV $300 \mathrm{MHz}$ NMR spectrometer. Mass spectral data were acquired by using 
a commercial LCQ ion trap mass spectrometer (ThermoFinnigan, SanJose, CA, USA) equipped with an ESI source or micromass Quatro LC triple quadrupole mass spectrometer for ESImass analysis. Column chromatography was performed with silica gel (Acme Synthetic Chemicals, India, 60-120 mesh). All the reagents were purchased from Sigma-Aldrich, St. Louis, USA unless otherwise stated. NP-40, antibiotics, and agarose were procured from Hi-media, India. HeLa, B16F10 and CHO cells were procured from the National Centre for Cell Sciences (NCCS), Pune, India. Cells were grown at $37{ }^{\circ} \mathrm{C}$ in Dulbecco's modified Eagle's medium (DMEM) with 10\% FBS in a humidified atmosphere containing $5 \% \mathrm{CO}_{2} / 95 \%$ air.

\section{Synthesis}

Detailed procedures for synthesizing the di-alkyl, di-hydroxy ethyl ammonium chloride based cationic amphiphiles are given below. Structures of all lipids and their synthetic intermediates were confirmed by ${ }^{1} \mathrm{H},{ }^{13} \mathrm{C}$ NMR and ESI-MS. Synthesis of cationic lipids was shown Fig. 2.

Steps A. Preparation of secondary amines (1A-5A, Fig. 2). Experimental procedure for the synthesis of intermediate $\mathbf{1 A}$ as representative procedure for preparation of secondary amines $(\mathbf{1 A}-\mathbf{5 A})$ is presented here. Briefly, in a $100 \mathrm{ml}$ round bottom flask, octadecylamine ( $1 \mathrm{~g}, 3.71 \mathrm{mmol})$, 1-bromooctadecane $(1.23 \mathrm{~g}, 3.71 \mathrm{mmol})$, and potassium carbonate $(2.5 \mathrm{~g}, 18.56$ $\mathrm{mmol}$ ) were dissolved in ethyl acetate. The reaction mixture was stirred at $80{ }^{\circ} \mathrm{C}$ for $16 \mathrm{~h}$. The reaction mixture was concentrated in rotary evaporator. The product is then recrystallized by using ethyl acetate $\left(1.7 \mathrm{~g}, 87 \%\right.$ yield, $R_{\mathrm{f}}=0.4,5: 95$ methanol :chloroform, v/v). The yield of 1A: $87 \%$; yield of 2A: $85 \%$; yield of 3A: $85 \%$; yield of $4 \mathrm{~A}$ : $79 \%$; yield of 5A: $80 \%$.

(i) N-Octadecyloctadecan-1-amine (intermediate 1A, Fig. 2). ${ }^{1} \mathrm{H}$ NMR $\left(\mathrm{CDCl}_{3}, 300 \mathrm{MHz}\right): \delta 0.83-0.89(\mathrm{t}, 6 \mathrm{H}), 1.19-1.32(\mathrm{~m}, 60 \mathrm{H})$; 1.39-1.42 (m, 4H), 1.71-1.76 (bs, 1H), 2.50-2.58 (m, 4H).

ESI-MS: $\mathrm{C}_{36} \mathrm{H}_{75} \mathrm{~N}$, calculated 521.59; found $522\left[\mathrm{M}^{+}\right]$.

(ii) $\mathrm{N}$-Hexadecyloctadecan-1-amine (intermediate $2 \mathrm{~A}$, Fig. 2 ). ${ }^{1} \mathrm{H}$ NMR ( $\left.\mathrm{CDCl}_{3}, 300 \mathrm{MHz}\right): \delta 0.82-0.89(\mathrm{t}, 6 \mathrm{H}), 1.21-1.30(\mathrm{~m}, 56 \mathrm{H})$; 1.39-1.43 (m, 4H), 1.73-1.80 (bs, $1 \mathrm{H}), 2.48-2.55$ (m, 4H).

ESI-MS: $\mathrm{C}_{34} \mathrm{H}_{71} \mathrm{~N}$, calculated 493.56; found $494\left[\mathrm{M}^{+}\right]$.

(iii) N-Tetradecyloctadecan-1-amine (intermediate 3A, Fig. 2). ${ }^{1} \mathrm{H}$ NMR $\left(\mathrm{CDCl}_{3}, 300 \mathrm{MHz}\right): \delta 0.85-0.91(\mathrm{t}, 6 \mathrm{H}), 1.21-1.30(\mathrm{~m}$, 52H); 1.41-1.46 (m, 4H), 1.73-1.82 (bs, 1H), 2.53-2.60 (m, 4H).

ESI-MS: $\mathrm{C}_{32} \mathrm{H}_{67} \mathrm{~N}$, calculated 465.53 ; found $466\left[\mathrm{M}^{+}\right]$.

(iv) N-Dodecyloctadecan-1-amine (intermediate 4A, Fig. 2). ${ }^{1} \mathrm{H}$ NMR ( $\left.\mathrm{CDCl}_{3}, 300 \mathrm{MHz}\right): \delta 0.81-0.89(\mathrm{t}, 6 \mathrm{H}), 1.15-1.23(\mathrm{~m}, 48 \mathrm{H})$; 1.38-1.44 (m, 4H), 1.70-1.78 (bs, 1H), 2.51-2.57 (m, 4H).

ESI-MS: $\mathrm{C}_{30} \mathrm{H}_{63} \mathrm{~N}$, calculated 437.50; found $438\left[\mathrm{M}^{+}\right]$.

(v) $\mathrm{N}$-Decyloctadecan-1-amine (intermediate 5A, Fig. 2). ${ }^{1} \mathrm{H} \mathrm{NMR}$ $\left(\mathrm{CDCl}_{3}, 300 \mathrm{MHz}\right): \delta 0.85-0.91(\mathrm{t}, 6 \mathrm{H}), 1.21-1.30(\mathrm{~m}, 44 \mathrm{H}) ; 1.41-$ $1.46(\mathrm{~m}, 4 \mathrm{H}), 1.73-1.82$ (bs, $1 \mathrm{H}), 2.53-2.60(\mathrm{~m}, 4 \mathrm{H})$.

ESI-MS: $\mathrm{C}_{28} \mathrm{H}_{59} \mathrm{~N}$, calculated 409.46; found $410\left[\mathrm{M}^{+}\right]$.

Step B. Synthesis of target Lip1818. Intermediate 1A prepared in Step A above $(1 \mathrm{~g}, 2.4 \mathrm{mmol}), \mathrm{K}_{2} \mathrm{CO}_{3}(1 \mathrm{~g}, 7.6 \mathrm{mmol})$, were dissolved in 2-chloroethanol in a sealed tube. The reaction mixture was stirred at $80{ }^{\circ} \mathrm{C}$ for $12 \mathrm{~h}$. The reaction mixture was concentrated in a rotary evaporator. The product is then recrystallized using ethyl acetate $\left(0.863 \mathrm{~g}, 71 \%\right.$ yield, $R_{\mathrm{f}}=0.2$, 5 : 95 methanol : chloroform, v/v). The yield of Lip1818: 71\%; yield of Lip1816: 69\%; yield of Lip1814: 70\%; yield of Lip1812: 67\%; yield of Lip1810: 65\%.

(i) 2,2'-(Dioctadecyl-L4-azanediyl)bis(ethan-1-ol), chloride salt (Lip1818, Fig. 2). ${ }^{1} \mathrm{H}$ NMR $\left(\mathrm{CDCl}_{3}, 300 \mathrm{MHz}\right): \delta 0.9(\mathrm{t}, 6 \mathrm{H}), 1.2(\mathrm{~m}$, $64 \mathrm{H}), 1.7(\mathrm{~m}, 4 \mathrm{H}), 3.5(\mathrm{~m}, 4 \mathrm{H}), 3.8(\mathrm{~m}, 4 \mathrm{H}), 4.2(\mathrm{~m}, 4 \mathrm{H})$.

ESI-MS: $\mathrm{C}_{40} \mathrm{H}_{84} \mathrm{NO}_{2}$, calculated 610.65; found $610\left[\mathrm{M}^{+}\right]$.

(ii) 2,2'-(Hexadecyl(octadecyl)- $\lambda^{4}$-azanediyl)bis(ethan-1-ol), chloride salt (Lip1816, Fig. 2). ${ }^{1} \mathrm{H} \mathrm{NMR}\left(\mathrm{CDCl}_{3}, 300 \mathrm{MHz}\right)$ : $\delta$ 0.83-0.92 (t, 6H), 1.12-1.40 (m, 56H); 1.61-1.72 (m, 4H), 3.38$3.48(\mathrm{~m}, 4 \mathrm{H}), 3.63-3.73(\mathrm{~m}, 4 \mathrm{H}), 4.04-4.15(\mathrm{~m}, 4 \mathrm{H}), 5.47-5.58$ (m, 2H).

ESI-MS: $\mathrm{C}_{38} \mathrm{H}_{80} \mathrm{NO}_{2}$, calculated 582.62; found $583\left[\mathrm{M}^{+}\right]$.

(iii) 2,2'-(Tetradecyl(octadecyl)- $\lambda^{4}$-azanediyl)bis(ethan-1-ol), chloride salt (Lip1814, Fig. 2). ${ }^{1} \mathrm{H}$ NMR $\left(\mathrm{CDCl}_{3}, 300 \mathrm{MHz}\right)$ : $\delta 0.85-0.93(\mathrm{t}, J=7.2 \mathrm{~Hz}, 6 \mathrm{H}), 1.17-1.41(\mathrm{~m}, 56 \mathrm{H}) ; 3.00-3.20(\mathrm{~m}$, $4 \mathrm{H}), 3.38-3.49(\mathrm{~m}, 2 \mathrm{H}), 3.65-3.73(\mathrm{~m}, 2 \mathrm{H}), 3.96-4.15(\mathrm{~m}, 4 \mathrm{H})$, 5.38-5.48 (bs, 2H).

ESI-MS: $\mathrm{C}_{36} \mathrm{H}_{76} \mathrm{NO}_{2}$, calculated 554.59; found $555\left[\mathrm{M}^{+}\right]$.

(iv) 2,2'-(Dodecyl(octadecyl)- $\lambda^{4}$-azanediyl)bis(ethan-1-ol), chloride salt (Lip1812, Fig. 2). ${ }^{1} \mathrm{H} \mathrm{NMR}\left(\mathrm{CDCl}_{3}, 300 \mathrm{MHz}\right): \delta 0.82-0.93$ $(\mathrm{t}, 6 \mathrm{H}), 1.16-1.41(\mathrm{~m}, 52 \mathrm{H}) ; 3.35-3.49(\mathrm{~m}, 4 \mathrm{H}), 3.63-3.73(\mathrm{~m}$, $4 \mathrm{H}), 4.03-4.15(\mathrm{~m}, 4 \mathrm{H}), 5.35-5.77(\mathrm{~m}, 2 \mathrm{H})$.

ESI-MS: $\mathrm{C}_{34} \mathrm{H}_{72} \mathrm{NO}_{2}$, calculated 526.56; found $527\left[\mathrm{M}^{+}\right]$.

(v) 2,2'-(Decyl(octadecyl)- $\lambda^{4}$-azanediyl)bis(ethan-1-ol), chloride salt (Lip1810, Fig. 2). ${ }^{1} \mathrm{H} \mathrm{NMR}\left(\mathrm{CDCl}_{3}, 500 \mathrm{MHz}\right): \delta 0.85-0.91(\mathrm{t}, J$ $=7.0 \mathrm{~Hz}, 6 \mathrm{H}), 1.19-1.32(\mathrm{~m}, 36 \mathrm{H}) ; 1.32-1.40$ (bs, 8H), 1.61-1.72 (bs, 4H), 3.39-3.47 (m, 4H), 3.64-3.71 (m, 4H), 4.05-4.13 (m, $4 \mathrm{H}), 5.47-5.54(\mathrm{~m}, 2 \mathrm{H})$.

ESI-MS: $\mathrm{C}_{32} \mathrm{H}_{68} \mathrm{NO}_{2}$, calculated 498.53; found $499\left[\mathrm{M}^{+}\right]$.

\section{Preparation of liposomes and plasmid DNA}

$1 \mathrm{mM}$ liposomes were prepared with $1: 1$ mole ratios of each lipid and cholesterol. Briefly, the cationic lipids and cholesterol in the appropriate mole ratios were dissolved in chloroform $(500 \mu \mathrm{l})$ in a glass vial. The solvent was removed with a thin flow of moisture-free nitrogen gas and the lipid film was kept for drying under high vacuum for $6 \mathrm{~h}$. $1 \mathrm{ml}$ of sterile deionized water was added to the vacuum dried lipid films and the mixtures were allowed to swell overnight. The vials were then vortexed for 2-3 minutes at room temperature to produce multilamellar vesicles (MLVs). MLVs were then sonicated initially in a water bath followed by an ice bath until clarity using a Branson 450 Sonifier at $100 \%$ duty cycle and $25 \mathrm{~W}$ output power to produce small unilamellar vesicles (SUVs). The p-CMV-SPORT- $\beta$-gal plasmid was amplified in DH5 $\alpha$-strain of Escherichia coli, isolated by alkaline lysis procedure and finally purified by PEG-8000 precipitation as described previously. ${ }^{29}$ The purity of plasmid was checked by $A_{260} / A_{280}$ ratio (around 1.9) and $1 \%$ agarose gel electrophoresis.

\section{DNA binding assay}

The DNA binding ability of the lipids were assessed by their gel retardation assay on a $1 \%$ agarose gel (pre-stained with ethidium bromide) across the varying lipid : DNA charge ratios 
of $8: 1$ to $1: 1$. pCMV- $\beta$-gal $(0.3 \mu \mathrm{g})$ was complexed with the varying amount of cationic lipids in a total volume of $30 \mu \mathrm{l}$ in HEPES buffer ( $\mathrm{pH} 7.4)$ and incubated at room temperature for 20-25 minutes. $4 \mu \mathrm{l}$ of $6 \times$ loading buffer $(0.25 \%$ bromophenol blue in $40 \%(\mathrm{w} / \mathrm{v})$ sucrose with sterile $\mathrm{H}_{2} \mathrm{O}$ ) was added to it and from the resulting solution $30 \mu \mathrm{l}$ was loaded on each well. The samples were electrophoresed at $80 \mathrm{~V}$ for 45 minutes and the DNA bands were visualized in the gel documentation unit.

\section{Zeta potential $(\xi)$ and size measurements}

The sizes and the zeta potentials (surface charges) of neat liposomes and lipoplexes with varying charge ratios $(8: 1-1: 1)$ were measured by photon correlation spectroscopy and electrophoretic mobility on a Zeta sizer $3000 \mathrm{HS}_{\mathrm{A}}$ (Malvern UK). The sizes and potentials of liposomes were measured in deionised water with a sample refractive index of 1.59 and a viscosity of 0.89. Liposomes of lipids, Lip1818-Lip1810 were complexed with DNA in plain DMEM for size and potential measurements of lipoplexes. The system was validated by using the $200 \mathrm{~nm}+$ $5 \mathrm{~nm}$ polystyrene polymer (Duke Scientific Corps. Palo Alto, CA). The diameters of liposomes and lipoplexes were calculated by using the automatic mode. The zeta potential was measured using the following parameters: viscosity, $0.89 \mathrm{cP}$; dielectric constant, 79; temperature, $25{ }^{\circ} \mathrm{C} ; \mathrm{F}(\mathrm{Ka}), 1.50$ (Smoluchowski); maximum voltage of the current, V. Using DTS0050 standard from Malvern, UK validated the system. All the size measurements were done 10 times in triplicate with the zero field correction and values represented as the average of triplicate measurements. The potentials were measured 10 times and represented as their average values as calculated by using the Smoluchowski approximation.

\section{Cryo-transmission electron microscopy of liposomes and lipoplexes}

The sample $(5 \mu \mathrm{l})$ was snap frozen using Tecnai Vitrobot (FEI Company, Hillsboro, OR, USA) in liquid ethane using holey carbon grids. Sample preparation was done at a constant temperature of $22{ }^{\circ} \mathrm{C}$. To relative humidity was maintained in the chamber to prevent solvent evaporation and changes in solvent concentration. The parameters used were as follows: blot time (s) - 1.0, blot force - 0 , wait time $(s)-1.0$, blot total - 1 , drain time $(s)-0.5$. Until imaging, the vitrified specimens were stored under liquid nitrogen. Imaging of the cryo-samples was done using Tecnai G2 Spirit Bio-TWIN Transmission Electron Microscope (FEI Company, Hillsboro, OR, USA) at $100 \mathrm{kV}$ with $4 \mathrm{k} \times 2 \mathrm{k}$ GatanOrius side mount CCD camera (Gatan, Pleasanton, CA).

\section{Small angle X-ray scattering (SAXS) measurements}

The Small Angle X-ray Scattering (SAXS) system was used to decipher the physical and structural interactions of liposomes of lipids, Lip1818-Lip1810 with the model membrane in solution. SAXS experiments were performed at Centre for Cellular and Molecular Biology, Hyderabad, India. The SAXS facility at CCMB is S3-MICRO Point-Focus system, Hecus X-ray systems, $\mathrm{GmbH}$ with 50 watt source power and $\mathrm{Cu}-\mathrm{K} \alpha(\lambda=1.54 \AA) \mathrm{X}$-rays.
The beam size at the sample is $50 \times 200 \mu \mathrm{m}^{2}$ with photon flux upto $2 \times 10^{8}$ photons per s. Pilatus $100 \mathrm{k}$ detector with a pixel size of $172 \times 172 \mu \mathrm{m}^{2}$ with a resolution range of $2000 \AA$ to $30 \AA$ was used. The sample-detector distance was maintained at 300 $\mathrm{mm}$ and $Q$-range of 0.003 to $0.6 \AA^{-1}$ that enables to cover a distance range of 2000 to $11 \AA$. Samples were filled into glass capillaries and flame-sealed. Scans were performed at $22{ }^{\circ} \mathrm{C}$ with an exposure time of $1 \mathrm{~h}$. Data was collected by using a Pilatus $100 \mathrm{k}$ detector. Diffraction intensity $v s$. $Q$ plots were obtained by radial integration of the $2 \mathrm{D}$ patterns by using the interactive data evaluating program FIT2D followed by ATSAS program suite.

\section{Transfection studies}

Cells were seeded at a density of 15000 cells (CHO, B16F10) per well in a 96-well plate $12-18 \mathrm{~h}$ before the transfection. $0.3 \mu \mathrm{g}$ of plasmid DNA was complexed with varying amounts of lipids (0.9-7.2 nmol) in complete DMEM/MEM medium (total volume made up to $100 \mu \mathrm{l}$ ) for 30 minutes. The lipid : DNA $( \pm)$ charge ratios were from $8: 1$ to $1: 1$ over these ranges of the lipids. The complexes were then added to the cells and incubated. The reporter gene activity was estimated between $36-48 \mathrm{~h}$. The cells were washed with PBS $(2 \times 100 \mu \mathrm{l})$ and lysed with $50 \mu \mathrm{l}$ lysis buffer [0.25 M Tris-HCl pH 8.0, 0.5\% NP40]. Care was taken to ensure complete lysis. The $\beta$-galactosidase activity per well was estimated by adding $50 \mu \mathrm{l}$ of $2 \times$-substrate solution $[1.33 \mathrm{mg}$ $\mathrm{ml}^{-1}$ of ONPG, 0.2 M sodium phosphate ( $\mathrm{pH}$ 7.3) and $2 \mathrm{mM}$ magnesium chloride] to the lysate in a 96-well plate. Absorption at $405 \mathrm{~nm}$ was converted to $\beta$-galactosidase units using a calibration curve constructed with the pure commercial $\beta$-galactosidase enzyme. The transfection experiments are the average of triplicate experiments with at least 3-4 values in each experiment.

\section{Cytotoxicity assay}

The cytotoxicity of lipids Lip1818-Lip1810 was evaluated in representative HeLa cells across the lipid:DNA charge ratios of 8 : 1-1 : 1 using MTT (3-(4,5-dimethylthiazol-2-yl)-2,5diphenyltetrazolium bromide) based reduction assay as described earlier. ${ }^{29}$ The cytotoxicity assay was performed in 96well plates by maintaining the same ratio of a number of cells to an amount of cationic lipid, as used in the previously described transfection experiments. Briefly, $4 \mathrm{~h}$ after the addition of lipoplexes, media was replaced with complete media and incubated for $24 \mathrm{~h}$. After $24 \mathrm{~h}$, MTT ( $5 \mathrm{mg} \mathrm{ml}^{-1}$ in PBS) was added to cells and incubated for $4 \mathrm{~h}$ at $37^{\circ} \mathrm{C}$. Results were expressed as percent viability $=\left[A_{540}(\right.$ treated cells $)-$ background $/ A_{540^{-}}$ (untreated cells) - background] $\times 100$.

\section{Cellular uptake studies by epifluorescence microscopy}

For fluorescence microscopy experiments, 10000 cells (HeLa) were seeded in each well of a 96-well plate (Corning Inc., Corning, NY) $12 \mathrm{~h}$ in $500 \mu \mathrm{l}$ of growth medium such that the well became $30-50 \%$ confluent at the time of transfection. Liposomes of lipids Lip1818-Lip1810 were complexed with pCMVa 5GFP $(0.3 \mu \mathrm{g}$ per well $)$ at $4: 1$ and $2: 1$ lipid : DNA charge ratio 
in a total volume of $100 \mu \mathrm{l}$ DMEM for 15-20 min. The complexes were then added to the cells. After $4 \mathrm{~h}$ incubation, media was replaced with complete media and incubated for 36-48 h. After incubation, cells were washed with PBS $(2 \times 100 \mu \mathrm{l})$ and fixed with $3.8 \%$ paraformaldehyde in PBS at room temperature for $10 \mathrm{~min}$. The green fluorescent cells were detected under an inverted fluorescence microscope (Nikon, Japan).

\section{Conflict of interest}

Authors declare no conflict of interest.

\section{Abbreviations}

$\begin{array}{ll}\text { DOPC } & \text { 1,2-Dioleoyl-sn-glycero-3-phosphocholine } \\ \text { DOPE } & \text { 1,2-Dioleoyl-sn-glycero-3-phosphoethanolamine } \\ \text { DOPS } & \text { 1,2-Dioleoyl-sn-glycero-3-phospho-L-serine }\end{array}$

\section{Acknowledgements}

PKV thanks Department of Biotechnology, India for Ramalingaswami Re-Entry fellowship, and DST for funding support (INT/SWISS/SNSFP-51/2015). AD thanks the Indian University Grant Commission for Junior Research Fellowship. MS thanks SERB-Fast track grant for the fellowship (SB/FT/CS-198/2013), Department of Science and Technology (DST). We thank Cellular Imaging Facility at inStem/NCBS, Bangalore and CSCR/ CMC, Vellore. We thank Dr Sankaranarayanan, Ms Rukmini Raju \& Mr Mallesh K, CCMB, Hyderabad, India for SAXS studies.

\section{Notes and references}

1 B. Draghici and M. A. Ilies, J. Med. Chem., 2015, 58, 40914130.

2 M. Jafari, M. Soltani, S. Naahidi, D. N. Karunaratne and P. Chen, Curr. Med. Chem., 2012, 19, 197-208.

3 M. Elsabahy, A. Nazarali and M. Foldvari, Curr. Drug Delivery, 2011, 8, 235-244.

4 K. K. Ewert, A. Zidovska, A. Ahmad, N. F. Bouxsein, H. M. Evans, C. S. McAllister, C. E. Samuel and C. R. Safinya, Top. Curr. Chem., 2010, 296, 191-226.

5 C. R. Safinya, K. K. Ewert, R. N. Majzoub and C. Leal, New J. Chem., 2014, 38, 5164-5172.

6 P. P. Karmali and A. Chaudhuri, Med. Res. Rev., 2007, 27, 696-722.

7 W. Li and F. C. Szoka Jr, Pharm. Res., 2007, 24, 438-449.

8 S. Bhattacharya and A. Bajaj, Chemical Commun., 2009, 46324656, DOI: 10.1039/b900666b.
9 V. V. Kumar, R. S. Singh and A. Chaudhuri, Curr. Med. Chem., 2003, 10, 1297-1306.

10 N. Monteiro, A. Martins, R. L. Reis and N. M. Neves, J. R. Soc., Interface, 2014, 11, 20140459.

11 S. Duangjit, B. Pamornpathomkul, P. Opanasopit, T. Rojanarata, Y. Obata, K. Takayama and T. Ngawhirunpat, Int. J. Nanomed., 2014, 9, 2005-2017.

12 Y. Zhao and L. Huang, Adv. Genet., 2014, 88, 13-36.

13 R. Mukthavaram, S. Marepally, M. Y. Venkata, G. N. Vegi, R. Sistla and A. Chaudhuri, Biomaterials, 2009, 30, 23692384.

14 R. P. Balasubramaniam, M. J. Bennett, A. M. Aberle, J. G. Malone, M. H. Nantz and R. W. Malone, Gene Ther., 1996, 3, 163-172.

15 B. G. Tenchov, L. Wang, R. Koynova and R. C. MacDonald, Biochim. Biophys. Acta, 2008, 1778, 2405-2412.

16 L. Wang and R. C. MacDonald, Gene Ther., 2004, 11, 13581362.

17 D. Zhi, S. Zhang, B. Wang, Y. Zhao, B. Yang and S. Yu, Bioconjugate Chem., 2010, 21, 563-577.

18 H. S. Kim, J. Moon, K. S. Kim, M. M. Choi, J. E. Lee, Y. Heo, D. H. Cho, D. O. Jang and Y. S. Park, Bioconjugate Chem., 2004, 15, 1095-1101.

19 M. H. Nantz, C. W. Dicus, B. Hilliard, S. Yellayi, S. Zou and J. G. Hecker, Mol. Pharmaceutics, 2010, 7, 786-794.

20 R. Koynova, B. Tenchov, L. Wang and R. C. Macdonald, Mol. Pharm., 2009, 6, 951-958.

21 R. Koynova, L. Wang and R. C. MacDonald, J. Phys. Chem. B, 2007, 111, 7786-7795.

22 R. Srinivas, S. Samanta and A. Chaudhuri, Chem. Soc. Rev., 2009, 38, 3326-3338.

23 R. R. Meka, S. Godeshala, S. Marepally, K. Thorat, H. K. R. Rachamalla, A. Dhayani, A. Hiwale, R. Banerjee, A. Chaudhuri and P. K. Vemula, RSC Adv., 2016, 6, 7784177848.

24 V. Chandrashekhar, M. Srujan, R. Prabhakar, R. C. Reddy, B. Sreedhar, K. K. Rentam, S. Kanjilal and A. Chaudhuri, Bioconjugate Chem., 2011, 22, 497-509.

25 R. S. Singh, K. Mukherjee, R. Banerjee, A. Chaudhuri, S. K. Hait, S. P. Moulik, Y. Ramadas, A. Vijayalakshmi and N. M. Rao, Chemistry, 2002, 8, 900-909.

26 J. Zimmerberg and M. M. Kozlov, Nat. Rev. Mol. Cell Biol., 2006, 7, 9-19.

27 K. Stiasny and F. X. Heinz, J. Virol., 2004, 78, 8536-8542.

28 Y. Onuki, Y. Obata, K. Kawano, H. Sano, R. Matsumoto, Y. Hayashi and K. Takayama, Mol. Pharmaceutics, 2016, 13, 369-378.

29 M. Srujan, V. Chandrashekhar, R. C. Reddy, R. Prabhakar, B. Sreedhar and A. Chaudhuri, Biomaterials, 2011, 32, 5231-5240. 